\begin{tabular}{|c|c|}
\hline Title & $\begin{array}{l}\text { Pharmaceutical immunoglobulins reduce neutrophil extracellular trap formation and ameliorate the development of } \\
\text { MPO-ANCA-associated vasculitis }\end{array}$ \\
\hline Author(s) & Uozumi, Ryo; Iguchi, Risa; Masuda, Sakiko; Nishibata, Y uka; Nakazawa, Daigo; Tomaru, Utano; Ishizu, A kihiro \\
\hline Citation & $\begin{array}{l}\text { Modern Rheumatology, 30(3), 544.550 } \\
\text { https://doi.org/10.1080/14397595.2019.1602292 }\end{array}$ \\
\hline Issue Date & 2019-04-12 \\
\hline Doc URL & http:/hdl.handle.net/2115/8182 \\
\hline Rights & $\begin{array}{l}\text { This is an A ccepted Manuscript of an article published by Taylor \& Francis in Modern Rheumatology on } 12 \text { A pr 2019, } \\
\text { available online: http://www.tandfonline.com/10.1080/14397595.2019.1602292. }\end{array}$ \\
\hline Type & article (author version) \\
\hline File Information & Ishizu2019.pdf \\
\hline
\end{tabular}

Instructions for use 


\title{
Pharmaceutical immunoglobulins reduce neutrophil extracellular trap formation and ameliorate the development of MPO-ANCA-associated vasculitis
}

Short title: Effect of IVIG-S on MPO-AAV

\author{
Ryo Uozumi ${ }^{1,2}$, Risa Iguchi ${ }^{1}$, Sakiko Masuda ${ }^{1}$, Yuka Nishibata ${ }^{1}$, Daigo Nakazawa ${ }^{3}$, \\ Utano Tomaru ${ }^{4}$, Akihiro Ishizu ${ }^{1}$
}

${ }^{1}$ Department of Medical Laboratory Science, Faculty of Health Sciences, Hokkaido University

${ }^{2}$ Division of Laboratory and Transfusion Medicine, Hokkaido University Hospital

${ }^{3}$ Division of Rheumatology, Endocrinology and Nephrology, Faculty of Medicine and Graduate School of Medicine, Hokkaido University

${ }^{4}$ Department of Pathology, Faculty of Medicine and Graduate School of Medicine, Hokkaido University

Correspondence: Akihiro Ishizu, MD, $\mathrm{PhD}$, Department of Medical Laboratory Science, Faculty of Health Sciences, Hokkaido University, Kita-12, Nishi-5, Kita-ku, Sapporo 0600812, Japan, TEL: +81-11-706-3385, FAX: +81-11-706-4916, E-mail: aishizu@med.hokudai.ac.jp 


\section{Abstract}

Objectives Intravenous immunoglobulin (IVIG) therapy is effective against some autoimmune diseases. Although its efficacy on peripheral neuropathy due to eosinophilic granulomatosis with polyangiitis - one of myeloperoxidase-antineutrophil cytoplasmic antibody (MPO-ANCA)-associated vasculitis (MPO-AAV) - has been established, that on other MPO-AAV remains undetermined. We examined the effects of pharmaceutical immunoglobulins on the formation of neutrophil extracellular traps (NETs) related to MPO-ANCA production and the development of MPO-AAV.

Methods Peripheral blood neutrophils from healthy volunteers were pretreated with 5 $\mathrm{mg} / \mathrm{ml}$ human sulfo-immunoglobulins (IVIG-S) and then exposed to $100 \mathrm{nM}$ phorbol myristate acetate (PMA). Thereafter, neutrophils were stained with SYTOX Green and then subjected to flow cytometry. Next, Wistar-Kyoto rats were given oral administration of $10 \mathrm{mg} / \mathrm{kg} /$ day propylthiouracil for 28 days and intraperitoneal (i.p.) injection of $1 \mu \mathrm{g}$ PMA on days 0 and 7 . These rats were divided into two groups: Group 1 with i.p. injection of $400 \mathrm{mg} / \mathrm{kg}$ IVIG-S on days 8-12 and Group 2 with i.p. injection of vehicle similarly. ANCA titers were chronologically determined by indirect immunofluorescence. On day 28, all rats were killed to examine NET formation in the peritoneum and the development of AAV.

Results IVIG-S significantly inhibited NET formation induced by PMA in vitro. NET amounts in the peritoneum in Group 1 were significantly smaller than in Group 2, and ANCA titers in Group 1 were significantly lower than in Group 2. The degree of pulmonary hemorrhage in Group 1 was also smaller than in Group 2.

Conclusion Pharmaceutical immunoglobulins reduce NET formation and ameliorate the development of MPO-AAV. 
Key words: anti-neutrophil cytoplasmic antibody (ANCA), ANCA-associated vasculitis, neutrophil extracellular traps (NETs), intravenous immunoglobulin (IVIG), animal model 


\section{Introduction}

Antineutrophil cytoplasmic antibody (ANCA)-associated vasculitis (AAV) is a necrotizing vasculitis that affects systemic small vessels accompanied by the presence of ANCA in the serum and includes microscopic polyangiitis (MPA), granulomatosis with polyangiitis (GPA), and eosinophilic granulomatosis with polyangiitis (EGPA) (1). The major target antigens of ANCAs are myeloperoxidase (MPO) and proteinase 3 (PR3). MPA affects preferentially the renal glomeruli, and the majority of the patients are positive for MPO-ANCA. GPA exhibits granulomatous inflammation with necrosis that usually involves the respiratory tract and simultaneously develops necrotizing small vessel vasculitis. Typical GPA patients are positive for PR3-ANCA but a considerable number of Japanese patients with GPA are positive for MPO-ANCA rather than PR3-ANCA (2). EGPA is an eosinophil-rich necrotizing vasculitis with granulomatous reaction that affects predominantly small- to medium-sized vessels. This disease is associated with asthma or allergic sinusitis. Approximately half of EGPA patients are positive for MPO-ANCA.

A combination of glucocorticoids and cyclophosphamide has been used as a standard remission induction therapy for MPO-AAV (3). This therapy has been proven to be effective with high remission induction rates of more than $90 \%$; however, the remaining patients do not respond to the therapy and approximately $20 \%$ patients relapse after remission. Recent international collaborative studies have determined new guidelines for remission induction therapy against MPO-AAV (4, 5). Furthermore, strenuous investigations into the pathogenesis of MPO-AAV have made several breakthroughs in this disease (6). Consequently, promising new therapeutic agents that modulate the immune system and target pathogenic B cells, $\mathrm{T}$ cells, cytokines, complement proteins, and humoral factors have been established (7). 
Pharmaceutical immunoglobulins are pooled preparation of $\gamma$-globulins from healthy blood donors and have been applied for the treatment of agammaglobulinemia (8), hypogammaglobulinemia (9), severe infections (10), and autoimmune diseases, including idiopathic thrombocytopenic purpura (11), Kawasaki disease (12), and Guillain-Barré syndrome (13). In addition, recent studies have demonstrated the efficacy of intravenous immunoglobulin (IVIG) therapy on EGPA, especially on vasculitis-associated peripheral neuropathy (14). Although the supplemental effects of $\gamma$-globulins are thought to be essential for agammaglobulinemia, hypogammaglobulinemia, and severe infections, it remains elusive how pharmaceutical immunoglobulins modulate the pathogenesis of autoimmune diseases. Moreover, it is also elusive in EGPA whether pharmaceutical immunoglobulins could act directly on peripheral nerves or improve neuronal function indirectly by acting on bystander affected small vessels. According to the nationwide epidemiological study of AAV, the prevalence of EGPA in AAV is approximately $10 \%$ in Japan (2). Therefore, it is worthy to know if pharmaceutical immunoglobulins are effective on other MPO-AAV as well as EGPA.

It has been considered that MPO-ANCA is a major pathogenic factor in MPO-AAV (6). For example, injection of either MPO-ANCA obtained by immunization of MPO-deficient mice with mouse MPO or splenocytes from these immunized mice into immunocompromised or wild-type mice can induce MPO-AAV. These findings clearly indicate the pathogenicity of MPO-ANCA (15).

More recent studies have demonstrated that neutrophil extracellular traps (NETs) are critically involved in the pathogenesis of MPO-AAV (16). NETs are web-like DNA decorated with antimicrobial proteins, including MPO, which are extruded from activated neutrophils (17). NETs can trap microorganisms by the web-like DNA and kill them using antimicrobial proteins. Although NET formation is 
regarded as an essential event in innate immunity, an excessive formation of NETs can induce vascular endothelial cell damage and thrombosis, resulting in the development of microvascular disorders (18). Actually, the disordered regulation of NETs has been suggested to be involved in the pathogenesis of autoimmune diseases, including MPO-AAV (19).

In the present study, we first determined that pharmaceutical immunoglobulins could inhibit NET formation induced by phorbol myristate acetate (PMA) in vitro and next examined the effects of pharmaceutical immunoglobulins in vivo using a rat model of MPO-AAV (20). 


\section{Materials and Methods}

\section{Human peripheral blood neutrophils}

Human peripheral blood polymorphonuclear cells (PMNs) were obtained from healthy volunteers by density-gradient centrifugation using Polymorphprep (Axis Shield, Dundee, Scotland). Because neutrophils are the most abundant cells in PMNs, PMNs were regarded as neutrophils in this study. During isolation, hemolysis process was omitted to avoid the nonspecific activation of neutrophils. This study was conducted with the permission of the Ethical Committee of the Faculty of Health Sciences, Hokkaido University (Permission No. 16-83).

\section{NET induction in vitro}

To induce NETs, human peripheral blood neutrophils $\left(1 \times 10^{6} / \mathrm{ml}\right)$ were exposed to $100 \mathrm{nM}$ PMA (Sigma-Aldrich, St. Louis, MO) for $3 \mathrm{~h}$ at $37^{\circ} \mathrm{C}$.

\section{Pretreatment with pharmaceutical immunoglobulins}

Thirty minutes before PMA stimulation, human peripheral blood neutrophils (1 $\times 10^{6} / \mathrm{ml}$ ) were treated with or without $5 \mathrm{mg} / \mathrm{ml}$ human sulfo-immunoglobulins (IVIG-S; Teijin, Tokyo, Japan) and incubated at $37^{\circ} \mathrm{C}$. The concentration is comparable to the effective blood concentration of IVIG-S in human medication (400 mg/kg body weight).

\section{NET detection}

NET formation was evaluated by flow cytometry (FCM) using SYTOX Green

(Life Technologies, Carlsbad, CA) and fluorescent staining with 4',6-diamidino-2-phenylindole (DAPI). The samples were allowed to react with a 
plasma membrane-impermeable DNA-binding dye SYTOX Green followed by analysis using Attune flow cytometer (Applied Biosystems, Foster City, CA ) as described previously (21). Simultaneously, NET formation was observed under fluorescent microscopy. For this purpose, peripheral blood neutrophils similarly treated in wells of four-well chamber slides (Thermo Fisher Scientific, Yokohama, Japan) $\left(1 \times 10^{6} / \mathrm{ml}\right)$ were washed with phosphate-buffered saline (PBS) followed by fixation with $4 \%$ paraformaldehyde for 15 min at room temperature. After washing with PBS, specimens were mounted using DAPI-containing mounting solution (Vector Laboratories, Burlingame, CA).

\section{Administration of IVIG-S to MPO-AAV model}

We employed the propylthiouracil (PTU) plus PMA-induced MPO-AAV model (20) in this study. Wistar-Kyoto (WKY) rats (4 weeks old, male, n=12) (Sankyo Laboratories, Sapporo, Japan) were given oral administration of $10 \mathrm{mg} / \mathrm{kg} / \mathrm{day}$ PTU for 28 days and intraperitoneal (i.p.) injection of $1 \mu \mathrm{g}$ PMA on days 0 and 7. These rats were divided into two groups, Group 1 (n=6) with i.p. injection of $400 \mathrm{mg} / \mathrm{kg}$ IVIG-S on days 8-12 and Group 2 (n=6) with i.p. injection of vehicle (PBS) similarly. Blood was obtained on days 0,14 , and 21 by tail cut for the chronological evaluation of ANCA titers. On day 28, all rats were killed to obtain whole blood and to examine NET formation in the peritoneum and the development of AAV. Experiment using rats were performed in accordance with the Guidelines for the Care and Use of Laboratory Animals in Hokkaido University (Permission No. 15-0034).

\section{Quantification of NETs in the peritoneum}

Peritoneal tissues obtained on day 28 were fixed with $10 \%$ formalin. Formalin-fixed paraffin-embedded (FFPE) tissues were sliced into $4 \mu \mathrm{m}$ sections and 
then deparaffinized with xylene. After antigen retrieval in an autoclave (at $121^{\circ} \mathrm{C}$ for 20 min) with Tris-EDTA buffer ( $\mathrm{pH} 9.0)$, the sections were soaked in Protein Block Serum-free (Dako, Glostrup, Denmark) for $10 \mathrm{~min}$ at room temperature to inhibit the nonspecific binding of antibodies. Antibodies to the NET marker, citrullinated histone 3 (Cit H3; rabbit polyclonal, 1:100 dilution; Abcam, Eugene, OR), were used as primary antibodies. After incubation with primary antibodies for $1 \mathrm{~h}$ at room temperature, the sections were washed with PBS. Secondary antibodies used were Alexa Fluor 594-conjugated goat anti-rabbit IgG H\&L at 1:500 dilution (Abcam). After incubation with secondary antibodies for $1 \mathrm{~h}$ at room temperature, the sections were washed with PBS and then mounted using DAPI-containing mounting solution (Vector Laboratories). The slides were observed under a fluorescence microscope. The Cit H3-positive DAPI-positive area was regarded as NET area that was determined by image analysis using ImageJ software.

\section{ANCA titers}

WKY rats (4 weeks old, male) were i.p. injected with $3 \%$ thioglycollate (Becton Dickinson, Tokyo, Japan). Three days later, rat neutrophils were collected by washing out the abdominal cavity. These cells were resuspended in RPMI 1640 medium supplemented with 5\% FBS $\left(1 \times 10^{6} / \mathrm{ml}\right)$, incubated in wells of four-well chamber slides (Thermo Fisher Scientific) for $30 \mathrm{~min}$ at $37^{\circ} \mathrm{C}$, air-dried, and then fixed with $100 \%$ ethanol for $10 \mathrm{~min}$ at room temperature. After washing with PBS, the cells were allowed to react with serially diluted rat sera $(1: 10,1: 20,1: 40,1: 80,1: 160$, and 1:320) for $60 \mathrm{~min}$ at room temperature. The cells were next allowed to react with 1:200 dilution of Alexa Fluor 488-conjugated goat anti-rat IgG (Thermo Fisher Scientific) for $30 \mathrm{~min}$ at room temperature. ANCA titers were determined as the highest dilution in which the majority of neutrophils exhibited positive staining. 
Secretion of lactoferrin from neutrophils treated with pharmaceutical immunoglobulins

Human peripheral blood neutrophils $\left(1 \times 10^{6} / \mathrm{ml}\right)$ were treated with or without $5 \mathrm{mg} / \mathrm{ml}$ IVIG-S at $37^{\circ} \mathrm{C}$. Thirty minutes later, supernatants were collected by centrifugation. The concentrations of lactoferrin in the supernatants were determined by enzyme-linked immunosorbent assay (ELISA) in the Japan Institute for the Control of Aging (Shizuoka, Japan).

\section{Statistical analysis}

For in vitro assays, Wilcoxon signed rank test was applied. For in vivo assays, Mann-Whitney $U$-test was applied for the comparison of two groups. $p<0.05$ was regarded as statistically significant. 


\section{Results}

\section{IVIG-S inhibited NET formation induced by PMA in vitro}

At first, we determined that IVIG-S at the concentration of clinical use (400 $\mathrm{mg} / \mathrm{kg}$ body weight) inhibited NET formation induced by PMA in vitro. FCM data demonstrated that the mean values of SYTOX Green intensity that represented the amounts of plasma membrane-bound extracellular DNA were significantly increased by PMA (100 nM for $3 \mathrm{~h}$ ); this indicated PMA-induced NET induction (Figure 1a). On the contrary, pretreatment of neutrophils with $5 \mathrm{mg} / \mathrm{ml}$ IVIG-S (in vitro concentration comparable to $400 \mathrm{mg} / \mathrm{kg}$ body weight) could significantly inhibit PMA-induced NET induction. The inhibitory effect was observed apparently in cases where PMA induced a considerable amount of NETs but not in cases where PMA induced a small amount of NETs. These findings suggest that IVIG-S inhibits NET formation depending on the increase in NETs induced by PMA.

The observation of cells under fluorescence microscope revealed corresponding results. As shown in Figure 1b, comet tail-like DNA staining was observed in neutrophils treated with PMA (100 $\mathrm{nM}$ for $3 \mathrm{~h}$ ), whereas it was not observed in neutrophils pretreated with $5 \mathrm{mg} / \mathrm{ml}$ IVIG-S.

\section{IVIG-S suppressed MPO-ANCA production and the development of vasculitic} lesions in a rat model of MPO-AAV

Next, we determined that $400 \mathrm{mg} / \mathrm{kg}$ IVIG-S suppressed MPO-ANCA production and the development of vasculitic lesions in a rat model of MPO-AAV. PTU plus PMA-induced MPO-AAV model rats were divided into two groups: Group 1 with i.p. injection of $400 \mathrm{mg} / \mathrm{kg}$ IVIG-S on days 8-12 and Group 2 with i.p. injection of vehicle (PBS) similarly. On day 28, the peritoneal tissues were obtained (Figure 2a) and 
then subjected to immunofluorescent staining for NETs (Figure 2b). The amounts of Cit H3-positive DNA in peritoneal tissues in Group 1 were significantly smaller than in Group 2 (Figure 2c).

ANCA titers were significantly lower in Group 1 than in Group 2 on days 21 and 28 (Figure 3). In addition, the development of pulmonary hemorrhage that represented vasculitic lesions in this model (Figure 4a) was significantly suppressed in Group 1 compared to Group 2 (Figure 4b). The collective findings suggested that IVIG-S at the concentration of clinical use can suppress NET formation in vivo as well as in vitro, MPO-ANCA production, and the development of MPO-AAV.

\section{IVIG-S induced lactoferrin secretion from neutrophils}

Recent studies have demonstrated that lactoferrin functions as an endogenous regulator of NET formation $(22,23)$. Therefore, we determined lactoferrin secretion from neutrophils before and after IVIG-S treatment. Results demonstrated that the concentrations of lactoferrin in culture supernatants of neutrophils were significantly increased after treatment by IVIG-S at the concentration of clinical use (Figure 5). 


\section{Discussion}

The efficacy of IVIG therapy on patients with severe infections and some autoimmune diseases has been established. Although the supplementation of $\gamma$-globulins seems to be significant for immunocompromised hosts, other mechanisms, including Fc-mediated inactivation of macrophages, consumption of activated complement proteins, neutralization of pathogenic autoantibodies by idiotype antibodies, and correction of cytokine imbalance, have been discussed as its effects on autoimmune diseases (24-26). In the present study, we additionally demonstrated that pharmaceutical immunoglobulins (IVIG-S) could suppress PMA-induced NET formation in vitro.

NETs are extracellular web-like DNA released from activated neutrophils, which are studded with antimicrobial proteins, such as MPO (17). Upon encounter with microbes, activated neutrophils undergo NETosis — cell death with NET formation — to trap and kill the microbes by NETs even after they died. Although NETs play an essential role in innate immunity, recent studies have demonstrated that an excessive formation of NETs can induce autoantibody production against the components of NETs, such as DNA (27) and MPO (20). Correspondingly, the decrease in NETs in peritoneal tissues by IVIG-S treatment resulted in the suppression of MPO-ANCA production and the subsequent development of vasculitic lesions-pulmonary hemorrhage - in the MPO-AAV induction model. PMA is a chemical but not biological substance. It has been demonstrated that ANCA and other serum factors are involved in NET formation in AAV patients $(19,28,29)$. Further studies are needed to demonstrate the inhibitory effect of IVIG-S on NET induction by those biological substances.

We hopefully discuss that IVIG-S can be applicable to MPO-AAV without a limitation to peripheral neuropathy in EGPA. In fact, a retrospective study has demonstrated the clinical benefit of IVIG as adjunctive therapy, with an acceptable 
tolerance profile, and thus supported its use in AAV patients with refractory or relapsing disease (30). Moreover, a clinical pilot study has demonstrated that IVIG therapy reduces the persistent disease activity of AAV (31). Although the inhibitory effect on NET formation might be linked to the suppression of innate immunity, it could be balanced by other promotional effects on the immune activity of IVIG-S.

Although the principal pathology of human MPO-AAV is NCGN, it was not apparent in the PTU plus PMA-induced MPO-AAV model in rats. The device for the exacerbation of NCGN in the rat model is needed to perform future studies.

It is currently undetermined in EGPA whether IVIG-S acts directly on peripheral nerves or improves neuronal function indirectly by acting on bystander affected small vessels. Because most EGPA patients have already been treated with glucocorticoids when administered with IVIG-S, we do not have information if their MPO-ANCA titers could decrease after IVIG-S treatment. We have observed that NETs are deposited in the affected vessels of EGPA patients (unpublished results) and NETs can contribute to vascular injury (18). Thus, it can be considered that IVIG-S prevents NET formation in small vessels and suppresses vasculitis, resulting in the improvement of peripheral neuropathy indirectly.

The precise mechanism by which IVIG-S suppresses NET formation remains unknown. Okubo et al. have recently demonstrated that lactoferrin immediately secreted from activated neutrophils can inhibit NET release (22); therefore, lactoferrin is regarded as an endogenous regulator of NET formation. In addition, Shida et al. have demonstrated that anti-lactoferrin antibodies - one of the "minor" ANCAs-are produced in some patients with EGPA and inhibit the effect of lactoferrin, resulting in the promotion of NET formation and disease activity (23). It has been demonstrated that the binding of immunoglobulins to Fc $\gamma$ receptors on neutrophils induces the immediate release of lactoferrin from neutrophils (32). Correspondingly, IVIG-S induces 
lactoferrin secretion from neutrophils. Lactoferrin released from IVIG-S-treated neutrophils might inhibit NET formation depending on the increase in NETs induced by PMA. Although further studies are needed, IVIG-S-induced lactoferrin secretion, at least in part, can be involved in the IVIG-S-mediated inhibition of NET formation from neutrophils.

The collective findings indicated that IVIG-S reduces NET formation and ameliorates the development of MPO-AAV. It is expected that future prospective clinical studies will prove the efficacy of IVIG therapy as one therapeutic option of treating patients with MPO-AAV.

\section{Acknowledgements}

This study was supported by a grant for Japan Research Committee of the Ministry of Health, Labour, and Welfare for Intractable Vasculitis (A.I.).

\section{Conflict of interest}

None. 


\section{References}

1 Jennette JC, Falk RJ, Bacon PA, Basu N, Cid MC, Ferrario F, et al. 2012 revised International Chapel Hill Consensus Conference Nomenclature of Vasculitides. Arthritis Rheum 2013;65:1-11.

2 Sada KE, Yamamura M, Harigai M, Fujii T, Dobashi H, Takasaki Y, et al. Classification and characteristics of Japanese patients with antineutrophil cytoplasmic antibody-associated vasculitis in a nationwide, prospective, inception cohort study. Arthritis Res Ther 2014;16:R101.

3 Ozaki S, Atsumi T, Hayashi T, Ishizu A, Kobayashi S, Kumagai S, et al. Severity-based treatment for Japanese patients with MPO-ANCA-associated vasculitis: the JMAAV study. Mod Rheumatol 2012;22:394-404.

4 Ntatsaki E, Carruthers D, Chakravarty K, D'Cruz D, Harper L, Jayne D, et al. BSR and BHPR guideline for the management of adults with ANCA-associated vasculitis. Rheumatology (Oxford) 2014;53:2306-9.

5 Yates M, Watts RA, Bajema IM, Cid MC, Crestani B, Hauser T, et al. EULAR/ERA-EDTA recommendations for the management of ANCA-associated vasculitis. Ann Rheum Dis 2016;75:1583-94.

6 Jennette JC, Falk RJ. Pathogenesis of antineutrophil cytoplasmic autoantibody-mediated disease. Nat Rev Rheumatol 2014;10:463-73.

7 Kallenberg CG. Advances in pathogenesis and treatment of ANCA-associated vasculitis. Discov Med 2014;18:195-201.

8 Shillitoe B, Gennery A. X-Linked Agammaglobulinaemia: Outcomes in the modern era. Clin Immunol 2017;183:54-62.

9 Makatsori M, Kiani-Alikhan S, Manson AL, Verma N, Leandro M, Gurugama NP, et al. Hypogammaglobulinaemia after rituximab treatment-incidence and 
outcomes. QJM 2014;107:821-8.

10 Shah PJ, Vakil N, Kabakov A. Role of intravenous immune globulin in streptococcal toxic shock syndrome and Clostridium difficile infection. Am J Health Syst Pharm 2015;72:1013-9.

11 Salama, A. Emerging drugs for immune thrombocytopenia (ITP). Expert Opin Emerg Drugs 2017;22:27-38.

12 Liu YC, Lin MT, Wang JK, Wu MH. State-of-the-art acute phase management of Kawasaki disease after 2017 scientific statement from the American Heart Association. Pediatr Neonatol 2018

13 Restrepo-Jimenez P, Rodriguez Y, Gonzalez P, Chang C, Gershwin ME, Anaya JM. The immunotherapy of Guillain-Barre syndrome. Expert Opin Biol Ther $2018 ; 18: 619-31$.

14 Koike H, Akiyama K, Saito T, Sobue G, Research Group for IVIg for EGPA/CSS in Japan. Intravenous immunoglobulin for chronic residual peripheral neuropathy in eosinophilic granulomatosis with polyangiitis (Churg-Strauss syndrome): a multicenter, double-blind trial. $\mathrm{J}$ Neurol 2015;262:752-9.

15 Xiao H, Heeringa P, Hu P, Liu Z, Zhao M, Aratani Y, et al. Antineutrophil cytoplasmic autoantibodies specific for myeloperoxidase cause glomerulonephritis and vasculitis in mice. J Clin Invest 2002;110:955-63.

16 Soderberg D, Segelmark M. Neutrophil extracellular traps in vasculitis, friend or foe? Curr Opin Rheumatol 2018;30:16-23.

17 Brinkmann V, Reichard U, Goosmann C, Fauler B, Uhlemann Y, Weiss DS, et al. Neutrophil extracellular traps kill bacteria. Science 2004;303:1532-5.

18 Dabrowska D, Jablonska E, Garley M, Sawicka-Powierza J, Nowak K. The Phenomenon of Neutrophil Extracellular Traps in Vascular Diseases. Arch 
Immunol Ther Exp (Warsz) 2018;66:273-81.

19 Nakazawa D, Shida H, Tomaru U, et al. Enhanced formation and disordered regulation of NETs in myeloperoxidase-ANCA-associated microscopic polyangiitis. J Am Soc Nephrol 2014;25:990-997.

20 Nakazawa D, Tomaru U, Suzuki A, Yoshida M, Nishio S, Atsumi T, et al. Abnormal conformation and impaired degradation of propylthiouracil-induced neutrophil extracellular traps: implications of disordered neutrophil extracellular traps in a rat model of myeloperoxidase antineutrophil cytoplasmic antibody-associated vasculitis. Arthritis Rheum 2012;64:3779-87.

21 Masuda S, Shimizu S, Matsuo J, Nishibata Y, Kusunoki Y, Hattanda F, et al. Measurement of NET formation in vitro and in vivo by flow cytometry. Cytometry A 2017;91:822-9.

22 Okubo K, Kamiya M, Urano Y, Nishi H, Herter JM, Mayadas T, et al. Lactoferrin Suppresses Neutrophil Extracellular Traps Release in Inflammation. EBioMedicine 2016;10:204-15.

23 Shida H, Nakazawa D, Tateyama Y, Miyoshi A, Kusunoki Y, Hattanda F, et al. The Presence of Anti-Lactoferrin Antibodies in a Subgroup of Eosinophilic Granulomatosis with Polyangiitis Patients and Their Possible Contribution to Enhancement of Neutrophil Extracellular Trap Formation. Front Immunol 2016;7:636.

24 Tha-In T, Bayry J, Metselaar HJ, Kaveri SV, Kwekkeboom J. Modulation of the cellular immune system by intravenous immunoglobulin. Trends Immunol 2008;29:608-15.

25 Baerenwaldt A, Biburger M, Nimmerjahn F. Mechanisms of action of intravenous immunoglobulins. Expert Rev Clin Immunol 2010;6:425-34.

26 Ballow $\mathrm{M}$. The $\mathrm{IgG}$ molecule as a biological immune response modifier: 
mechanisms of action of intravenous immune serum globulin in autoimmune and inflammatory disorders. J Allergy Clin Immunol 2011;127:315-23.

27 Hakkim A, Furnrohr BG, Amann K, Laube B, Abed UA, Brinkmann V, et al. Impairment of neutrophil extracellular trap degradation is associated with lupus nephritis. Proc Natl Acad Sci USA 2010;107:9813-8.

28 Kessenbrock K, Krumbholz M, Schonermarck U, Back W, Gross WL, Werb Z, et al. Netting neutrophils in autoimmune small-vessel vasculitis. Nat Med $2009 ; 15: 623-5$.

29 Kraaij T, Kamerling SWA, van Dam LS, Bakker JA, Bajema IM, Page T, et al. Excessive neutrophil extracellular trap formation in ANCA-associated vasculitis is independent of ANCA. Kidney Int 2018;94:139-49.

30 Crickx E, Machelart I, Lazaro E, Kahn JE, Cohen-Aubart F, Martin T, et al. Intravenous immunoglobulin as an immunomodulating agent in antineutrophil cytoplasmic antibody-associated vasculitides: a French nationwide study of ninety-two patients. Arthritis Rheumatol 2016;68:702-12.

31 Jayne DR, Chapel H, Adu D, Misbah S, O'Donoghue D, Scott D, et al. Intravenous immunoglobulin for ANCA-associated systemic vasculitis with persistent disease activity. QJM 2000;93:433-9.

32 Teeling JL, De Groot ER, Eerenberg AJ, Bleeker WK, Van Mierlo G, Aarden LA, et al. Human intravenous immunoglobulin (IVIG) preparations degranulate human neutrophils in vitro. Clin Exp Immunol 1998;114:264-70. 


\section{Figure legends}

\section{Figure 1. Inhibition of PMA-induced NET formation by IVIG-S}

(a) Peripheral blood neutrophils from healthy volunteers $(n=6)$ were pretreated with 5 $\mathrm{mg} / \mathrm{ml} \mathrm{IVIG-S}$ for $30 \mathrm{~min}$ at $37^{\circ} \mathrm{C}$ and then exposed to $100 \mathrm{nM}$ PMA for $3 \mathrm{~h}$ at $37^{\circ} \mathrm{C}$. Thereafter, neutrophils were stained with SYTOX Green and then subjected to FCM. The mean fluorescent intensities of SYTOX Green are plotted. ${ }^{*} p<0.05$. (b) Neutrophils similarly treated in wells of four-well chamber slides $\left(1 \times 10^{6} / \mathrm{ml}\right)$ were washed with PBS followed by fixation with $4 \%$ paraformaldehyde for $15 \mathrm{~min}$ at room temperature. After washing with PBS, specimens were mounted using DAPI-containing mounting solution. Representative microphotographs are shown. Bar, $50 \mu \mathrm{m}$.

\section{Figure 2. Decrease in peritoneal NETs in IVIG-S-administered MPO-AAV model}

WKY rats (4 weeks old, male, $\mathrm{n}=12$ ) were given oral administration of $10 \mathrm{mg} / \mathrm{kg} / \mathrm{day}$ PTU for 28 days and i.p. injection of $1 \mu \mathrm{g}$ PMA on days 0 and 7. These rats were divided into two groups: Group 1 with i.p. injection of $400 \mathrm{mg} / \mathrm{kg}$ IVIG-S on days 8-12 and Group 2 with i.p. injection of vehicle similarly. On day 28, all rats were killed and peritoneal tissues were obtained. FFPE sections of peritoneal tissues were subjected to immunofluorescent staining for $\mathrm{Cit} \mathrm{H} 3$ and then mounted using DAPI-containing mounting solution. (a) Representative histology of the peritoneal tissues. Hematoxylin and eosin staining $(\times 100)$. Bar, $100 \mu \mathrm{m}$. (b) Microphotographs of the high power field $(\times 100)$ that exhibited the most abundant formation of NETs are shown. Red, Cit H3; blue, DNA. Bar, $100 \mu \mathrm{m}$. (c) NET areas determined as pixel counts of the purple area are plotted.

Figure 3. Suppressed ANCA production in IVIG-S-administered MPO-AAV model 
ANCA titers were chronologically determined by indirect immunofluorescence as the highest dilution in which the majority of neutrophils exhibited positive staining. $* p<0.05, * * p<0.01$.

Figure 4. Reduced pulmonary hemorrhage in IVIG-S-administered MPO-AAV model

(a) Representative microphotograph of pulmonary hemorrhage observed in Group 2 (original magnification, $\times 100$ ). (b) Number of hemorrhagic foci in the pulmonary sections counted under a microscope. ${ }^{*} p<0.05$.

\section{Figure 5. Lactoferrin secretion from neutrophils induced by IVIG-S}

Human peripheral blood neutrophils $\left(1 \times 10^{6} / \mathrm{ml}\right)$ were treated with or without $5 \mathrm{mg} / \mathrm{ml}$ IVIG-S at $37^{\circ} \mathrm{C}$. Thirty minutes later, supernatants were collected by centrifugation. The concentrations of lactoferrin in the supernatants were determined by ELISA. $* * p<0.01$. 
Figure 1

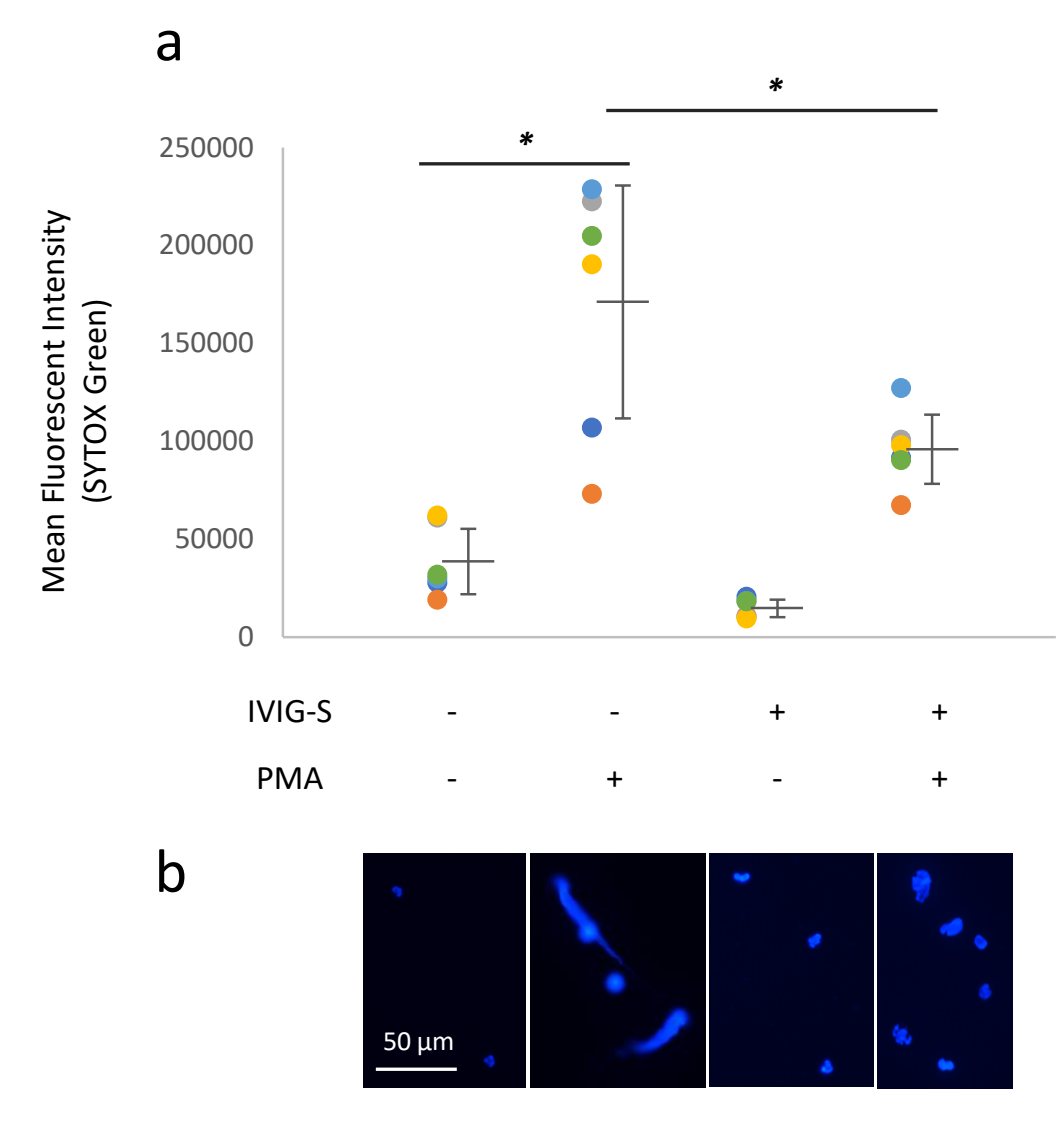




\section{Figure 2}

Non-treated

a

b

Group 1

(IVIG-S)



Group 1 (IVIG-S)




Group 2 (PBS)

DNA Cit H3
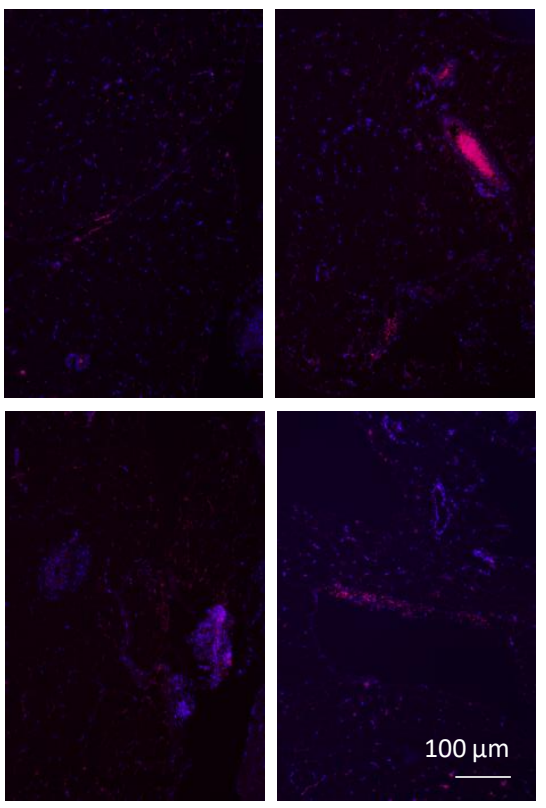

Group
(PBS)

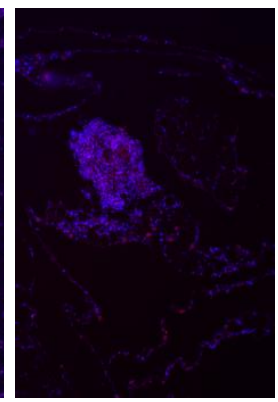

C

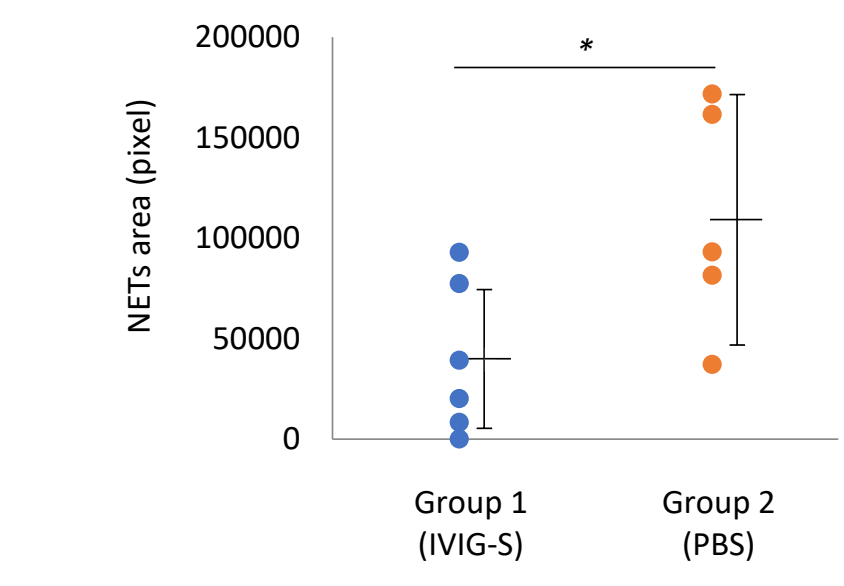


200

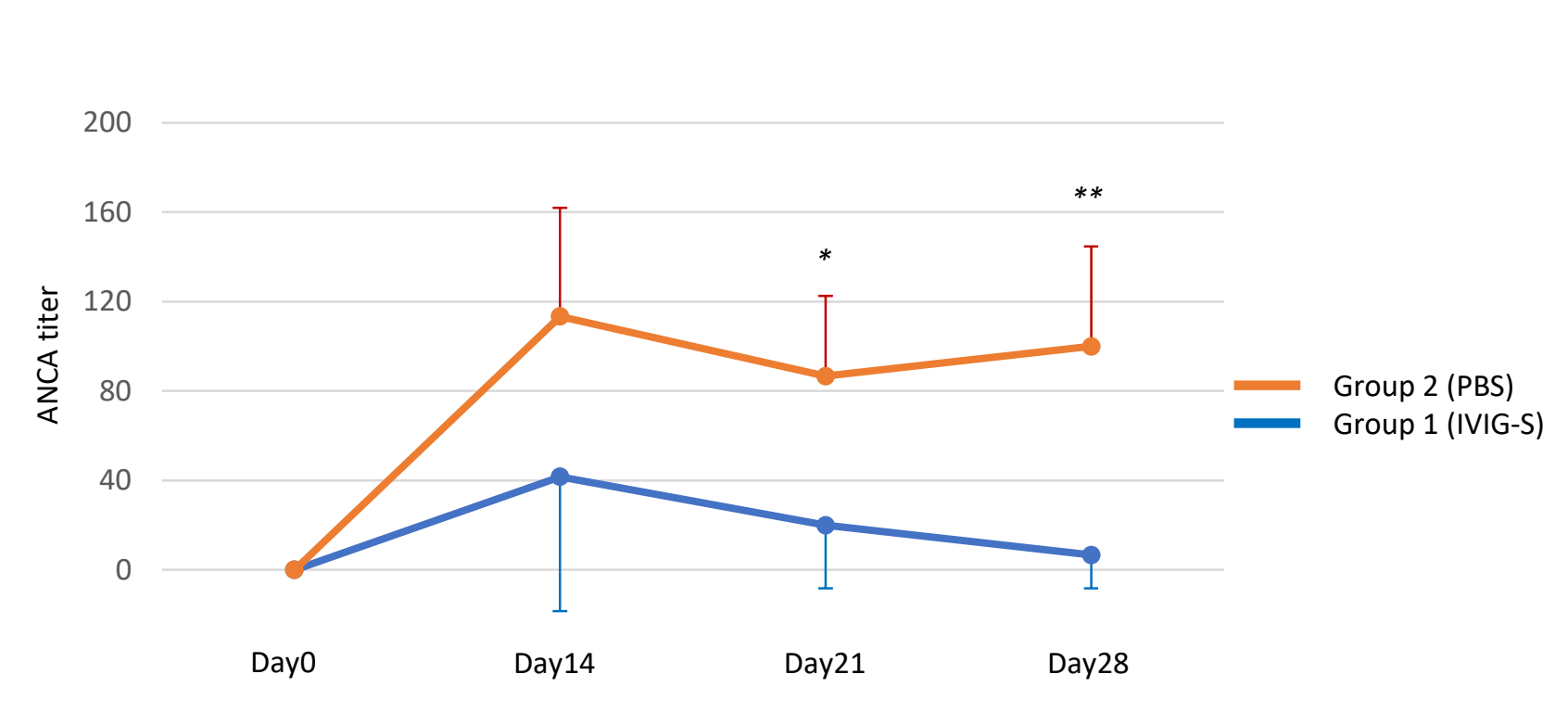

\section{Figure 3}




\section{Figure 4}

Figure 5

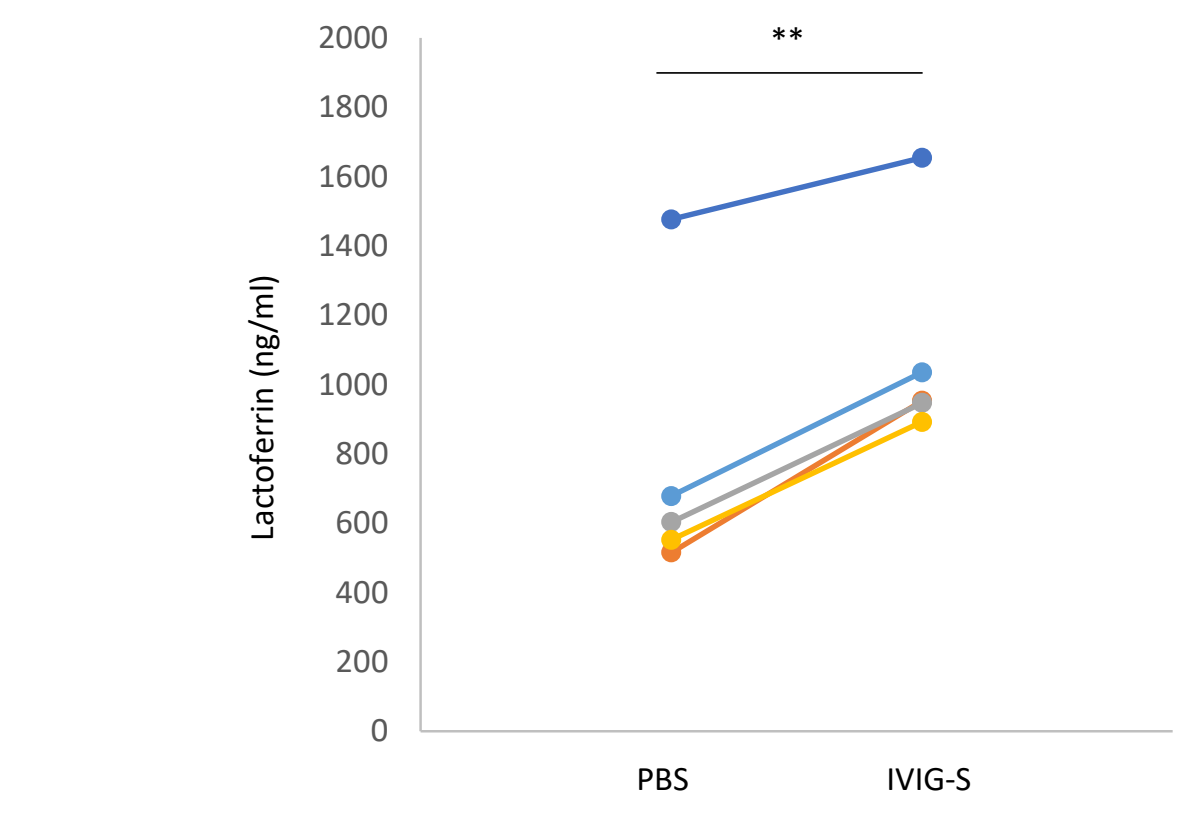

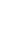

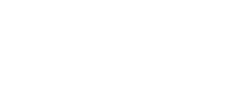
PBS 